\title{
PARADIGMAS EDUCATIVOS PARA UNA METODOLOGÍA GLOBAL EN LA ENSEÑANZA
}

\author{
Reina Castellanos Vega \\ Universidad de Zaragoza \\ Facultad de educación \\ Fabio Avilés Acero \\ Universidad de Zaragoza \\ Facultad de educación \\ Alberto Cazaña Garcés \\ Universidad de Zaragoza \\ Facultad de educación \\ Atila Gabarrús Szöllösi \\ Universidad de Zaragoza \\ Facultad de educación
}

Recepción Artículo: 20 mayo 2021

Admisión Evaluación: 20 mayo 2021

Informe Evaluador 1: 28 mayo 2021

Informe Evaluador 2: 29 mayo 2021

Aprobación Publicación: 02 junio 2021

\section{RESUMEN}

Como docentes nos encontramos con alumnos con diferentes características: incorporación tardía de alumnos, provenientes de familias desestructuradas donde escasean normas, con carencias en hábitos de trabajo y de estudio, alumnos con Necesidades Específicas de Apoyo Educativo (NEAE). Todo ello puede crear en el aula una falta de convivencia, de empatía y de cohesión de grupo. Este estudio se fundamenta principalmente en tres tipos de paradigmas: el conductista, el cognitivo-humanista y el cognitivo-sociocultural. La realización de esta propuesta no es una dinámica puntual, ya que pensamos que este tipo de solución sería menos efectiva puesto que no hay una persistencia en el tiempo ni sobre los alumnos, sino que optamos por un método pedagógico anual o trimestral. El objetivo de este estudio es elaborar una propuesta desarrollando el trabajo de modificación de conducta, la cohesión grupal, el incremento total de las capacidades del alumnado, fomentar un ambiente agradable en la clase para motivar a los estudiantes, así como la creación de una relación cercana y de confianza entre el profesor y su clase y realizar una primera aproximación para llevar a cabo en futuras investigaciones el método propuesto. Es una investigación etnográfica centrada en los alumnos de primer cuatrimestre de magisterio de 


\section{PARADIGMAS EDUCATIVOS PARA UNA METOdOLOGÍA GLOBAL EN LA ENSEÑANZA}

educación primaria. Según resultados, en la clase se presentan grupos cerrados con alguna conexión a través de algún compañero, además, hay alumnos que son rechazados por su forma de vincularse 0 por su discapacidad. Siendo necesario trabajar esta metodología en todos los niveles educativos, vista los datos nos indican que los futuros profesores de primaria presentan y viven esta situación. Entre docentes y discentes consideramos mejorar la enseñanza utilizando una metodología que ofrezca seguimiento no solo a nivel de conocimientos sino de cohesión de grupo y de bienestar en el aula.

Palabras clave: paradigma; modificación de conducta; cohesión de grupo; sociograma; método pedagógico

\section{ABSTRACT}

Educational paradigms for a global methodology in teaching. As teachers we find students with different characteristics: late incorporation of students, coming from unstructured families where standards are scarce, with deficiencies in work and study habits, students with Specific Needs of Educational Support (NEAE). All this can create in the classroom a lack of coexistence, empathy and group cohesion. This study is based mainly on three types of paradigms: the behaviorist, the cognitive-humanist and the cognitive-sociocultural. The implementation of this proposal is not a specific dynamic, since we believe that this type of solution would be less effective since there is no persistence in time or on the students, but we opt for an annual or quarterly pedagogical method. The objective of this study is to make a first approach to carry out in future investigations the proposed method, developing the work of behavior modification, group cohesion, the total development of the capacities of the students, encourage a pleasant atmosphere in the classroom to motivate students, as well as the creation of a close and trusting relationship between the teacher and his or her class. It is an ethnographic research focused on first-semester primary school teachers. According to the results, in the class there are closed groups with some connection through a partner, in addition, there are students who are rejected because of their way of bonding or because of their disability. Since it is necessary to work on this methodology at all levels of education, the data show that future primary school teachers present and experience this situation. Among teachers and students we believe that improving teaching using a methodology that offers follow-up not only at the level of knowledge but of group cohesion and well-being in the classroom.

Keywords: paradigm; behavior modification; group cohesion; sociogram; pedagogical method

\section{INTRODUCCIÓN}

Hay situaciones en las que como docentes tenemos que enfrentarnos a conductas inadecuadas, mas no agresivas o increpantes y pueden ser normalizadas por el resto de los compañeros, creando un ambiente poco saludable para una buena educación. Las conductas inadecuadas de los alumnos pueden ser causa de la convivencia con familias desestructuradas y/o hogares con falta de normas, otros, con alguna discapacidad o con Necesidades Específicas de Apoyo Educativo (NEAE) esto hace que los estudiantes no sepan cómo gestionar su frustración. Debido a todos estos factores, en el aula se puede presentar una falta de empatía, de convivencia y de cohesión de grupo. Para resolver todos estos problemas, vamos a recurrir a una metodología educativa que abarca todo el curso escolar, convirtiéndose en el sistema a seguir del profesor-tutor a lo largo de este.

\section{MARCO TEÓRICO}

Los fundamentos teóricos de este proyecto anual son varios, siendo el paradigma conductista de Pavlov y Skinner (Morales y Irigoyen, 2017), el paradigma cognitivo-humanista de Rogers (Lima, Barbosa y Peixoto, 2018) y por último el paradigma sociocultural o sociohistórico de Vygotsky 
(Villamizar, 2017). Haremos una combinación de estas tres teorías educativas para crear una amalgama que recoja los fundamentos teóricos que solventará la situación de la clase.

\section{Paradigma conductista}

Comenzamos con el paradigma conductista, teoría construida por el psicólogo y pedagogo Burrhus Frederic Skinner, y por el fisiólogo Ivan Pávlov (Morales y Irigoyen, 2017). Esta teoría tiene como concepto de enseñanza la depositación de información, significando esto que es el profesor el que contiene todos los conocimientos, y los lanza a su alumnado de manera unidireccional. En esta teoría nos encontramos con un alumnado que juega un papel pasivo, lo que significa que este sólo recibe la información, sin participar en el desarrollo de la clase, a excepción de preguntas/dudas. En cuanto al papel del profesorado, nos topamos con un ingeniero constructor $\mathrm{e}$ instructor, es decir, un profesor que utiliza los estímulos en forma de recompensas para motivar al alumno. Dentro de este paradigma nos encontramos con dos tipos de aprendizaje, comprendiendo a este de una manera conductual: condicionamiento clásico y operante (Morales y Irigoyen, 2017) .

El primero de ellos es el condicionamiento clásico: es un tipo de aprendizaje desarrollado por Ivan Pávlov, en el que un organismo aprende a transferir una respuesta natural ante un estímulo a otro estímulo previamente neutro, que después se convierte en condicionado. Para que este condicionamiento pueda ser llevado a cabo correctamente, hay que cumplir con una serie de requisitos. Estos requisitos son: "1. El orden de presentación de los estímulos, no debe de hacerse de forma simultánea, ni mucho después. 2. El tiempo. Deben mediar segundos entre el Estímulo Neutro (EN) y el Estímulo Incondicionado (EI). 3. La secuencia debe repetirse varias veces (EN-EI)." Castellanos (2020, p. 69).

Según Castellanos (2020), uno de los conceptos básicos del condicionamiento clásico es el refuerzo, en el cual nos encontramos con tres ítems: "1. Es el apareamiento de los Estímulos Condicionados (EC) con los Estímulos Incondicionados (EI) 2. Si no se introduce cada cierto tiempo el El, las RC se van debilitando hasta llegar a desaparecer (extinción). 3. Recuperación espontáneavolver a emitir la respuesta que parecía ya desaparecida". También son considerados por Castellanos (2020) como conceptos básicos del condicionamiento clásico la Generalización y Discriminación. "Generalización: Respuesta a la similitud de los estímulos (otros sonidos) y discriminación: Responde a las diferencias (solo a la campana)" (Castellanos, 2020, p. 70)

El segundo de ellos es el Condicionamiento operante: podemos comprender el condicionamiento operante como "un proceso a través del cual aprendemos a dar respuestas adecuadas para obtener algún beneficio o evitar algo que nos desagrada" (Castellanos, 2020, p. 72) Encontrándonos de este modo con dos tipos de consecuencias: Las consecuencias gratificantes, las cuales tendrán un papel de refuerzo, puesto que se intentarán repetir, y las consecuencias desagradables 0 aversivas, que serán aplicadas como castigo teniendo un efecto reductor 0 debilitador de la conducta (Bertrán, 2019).

\section{Paradigma cognitivo- humanista}

La siguiente teoría que vamos a contextualizar dentro de este marco teórico es la CognitivoHumanista. Esta se encuentra dentro de las teorías cognitivas que según Francia (2020) "El cognitivismo, apareció en los años sesenta con las investigaciones de U. Neisser, que hizo la primera formulación teórica en Psicología Cognitiva (1967), se ha expandido con las investigaciones de A. Collins, G.A. Miller, D. Norman, G. Mandler, D.E. Rumelhart, J.S. Bruner" (Francia, 2020, p. 1) de acuerdo con esta misma psicóloga podemos decir que el cognitivismo no se conforma tanto como una escuela psicológica, sino una orientación que toma de diferentes corrientes y escuelas psicológicas, la cual se encuentra totalmente opuesta a la teoría conductista en su base. Como ya 


\section{PARADIGMAS EDUCATIVOS PARA UNA METOdOLOGÍA GLOBAL EN LA ENSEÑANZA}

aclaramos con anterioridad, nos centraremos en la teoría cognitivo-humanista, que coincidiendo con Fingermann (2012), podemos decir que esta teoría fue desarrollada por Carl Rogers (19021987), en conjunto con Abraham Maslow.

Rogers se basó en la comunicación y empatía humana en todas sus teorías, por supuesto también en la educación. La teoría cognitivo humanista se basa en la bondad intrínseca del ser humano de Rosseau, en el deseo de la adquisición de conocimientos como parte del ser humano, en la percepción totalizadora de la Gestalt, nacida en la Alemania del siglo XX, en la confianza del conocimiento basado en la experiencia, en algunas aportaciones tomadas del psicoanálisis como el relativismo cultural y científico, y por último en el existencialismo de Gabriel Marcel y Soren Aabye Kierkegaard. Rogers proclamó la necesidad de educar de forma libre y pragmática, teniendo un maestro que orienta, ayuda, de caminos a seguir, que escuche a su alumnado, participando en el grupo clase como uno más, facilitando sus aportaciones y que aprende también de las conclusiones de sus alumnos, evitando cualquier tipo de imposición de conductas, puesto que lo que busca es que salgan de manera espontánea, intuitiva, respetuosa y natural hacia la perfección de las mismas, puesto que Rogers defiende que de este modo es como se expresarán las potencialidades del alumnado a medida que vaya cambiando su existencia así como la del entorno.

Nos encontramos dentro de esta teoría con un concepto de enseñanza flexible y abierto, puesto que esta teoría se encuentra basada en la madurez y el desarrollo personal de cada alumno, adquiriendo los conocimientos en base a estas dos variables. Dictando así una educación personalizada y basada en los intereses del alumno, puesto que este solo aprenderá lo que realmente le importe o considere significativo, siendo este el responsable de su autoevaluación, aclarando así que el papel que tiene el alumnado dentro de esta teoría es completamente autónomo. Por todos estos aspectos podemos decir que en el paradigma cognitivo humanista contamos con un concepto de aprendizaje basado en el desarrollo personal del alumnado. En cuanto a las estrategias que esta teoría utiliza para el correcto desarrollo de los periodos educativos podemos observar el uso de la división en grupos del alumnado, la facilitación de los recursos por parte del profesor, el trabajo por proyectos y las tutorías entre los propios compañeros.

\section{Paradigma sociocultural}

El último paradigma en el que nos hemos basado es la teoría sociocultural o sociohistórica de Vygotsky y Bandura. Como podemos ver en el escrito de Vielma y Salas (2000), el objetivo principal de esta teoría es dar una explicación al desarrollo humano acorde con las concepciones dinámicas y transformadoras que encontramos en el contexto genético-sociocultural. "Bandura nos propone un paradigma que mantiene preferencia por la investigación focalizada en el desarrollo humano, sólo entendible por la acción del aprendizaje dentro de contextos sociales a través de modelos en situaciones reales y simbólicas" (Vielma y Salas, 2000, P. 34) Centrando su énfasis en los procesos cognitivos autorreguladores y autorreflexivos. Por otro lado, nos encontramos con las palabras que el mismo Vygotsky dijo, "La educación, por su parte, puede ser definida como el desarrollo artificial del niño. La educación es el dominio ingenioso de los procesos naturales del desarrollo. La educación no sólo influye sobre unos u otros procesos del desarrollo, sino que reestructura, de la manera más esencial, todas las funciones de la conducta" (Baquero, 1996, p. 105).

En este paradigma podemos observar un concepto de enseñanza basado en el proceso de internalización sobre la realidad (entorno), teniendo como concepto de aprendizaje basado en la instrucción, lo que quiere decir que la información que precisa el alumnado se obtiene a través de las relaciones con su entorno. En cuanto al papel del alumno, nos encontramos con un alumnado socio-activo, lo que significa que el alumno se encuentra continuamente dentro de un sistema 
social, en el que participa activamente, tanto como receptor de información, así como de generador de la misma.

En este paradigma nos encontramos con un profesor que hace de mediador entre el alumno y el conocimiento, siendo este el experto que puede guiar al alumnado. La estrategia más usada por esta teoría es lo que se denomina "Andamiaje", coincidiendo con Carrillo, Rivera y Benjamín (2010) concebimos el andamiaje como la prestación de apoyo o ayuda, en una situación de cooperación, de la persona más hábil a la persona que es menos hábil en un campo del conocimiento, para que el segundo pueda llegar a completar la tarea. Conforme la persona menos hábil vaya dando muestras de ir alcanzando las distintas situaciones, el experto va retirando su apoyo hasta que pueda retirar la ayuda por completo. Así como también se subdivide al grupo clase, en varios grupos de trabajo, fomentando la cooperación, el compañerismo, y el propio andamiaje.

Por último, el sistema de evaluación con el que cuenta este paradigma es el de la determinación del nivel de desarrollo, el cual se mide desde el Nivel de Desarrollo Real (NDR), el cual es el nivel de adquisición de conocimientos que tiene el alumno en el momento en el que se comienza la adquisición del conocimiento, y el nivel de desarrollo próximo (NDP), el cual es el nivel de adquisición de conocimientos al cual el alumno puede llegar a alcanzar tras la recogida de información.

A continuación, vamos a hablar de la modificación de la conducta en el aula. Cuando hablamos de modificación de conducta en el aula, coincidiendo con González - Pérez y Criado (2010) entendemos el cambio de conducta como "Conjunto de técnicas muy diferentes que se aplican a la solución de los problemas de conducta en los campos de la educación, la psicología clínica y la rehabilitación" (González - Pérez y Criado, 2010, p. 77).

Esta modificación de la conducta se basa en las teorías del condicionamiento clásico y operante, en el aprendizaje social y en la psicología cognitiva. Al hablar de la modificación de conducta en el aula tenemos que hablar de cuatro pilares fundamentales que son: 1. El profesor como agente de modificación y cambio de conducta. En este modelo tenemos a un profesor que ha de ser un referente significativo para los alumnos, debiendo estructurar el medio físico y social, fomentando entornos educativos que permitan experimentar relaciones seguras y en el que pueda desarrollar los estilos personales democráticos, tolerantes y comprensivos de cada uno de los alumnos.

Según Castellanos (2020) para realizar una intervención tenemos que poder reconocer alguno de los siguientes puntos: Reconocer un comportamiento perjudicial para la integridad física, para la adaptación o interfiere en el progreso académico. También reconocer cuando un comportamiento daña a otras personas 0 a sus pertenencias, así como cuando afecta a la eficacia profesional 0 a la estabilidad emocional de cualquier alumno o profesor. 2. Evaluación conductual, es "un proceso clave para determinar el éxito de cualquier técnica de modificación de conducta" (González - Pérez y Criado, 2010, p. 81). Puesto que con esta evaluación podemos identificar las conductas problemáticas, las variables que están controlando su aparición y los objetivos que se quieren lograr con la intervención. Pudiendo de este modo definir el por qué se produce esa actuación, así como definir cuáles son sus problemas.

El proceso que hemos de llevar para poder efectuar una evaluación conductual de manera exitosa es el siguiente: Primero hemos de definir la operativa de la conducta, para un posterior análisis funcional de la conducta. Este análisis está basado en el modelo secuencial integrativo de Kanfer, Phillips y Fernández-Ballesteros, desarrollado a partir de la observación, el cual según Aragón y Silva (2008) se basa en los estímulos antecedentes (E) que provocan en el presente las respuestas en las personas y terminan en consecuencias producidas hacia el medio ambiente.

También este modelo une lo cognoscitivo con lo conductual, ya que los antecedentes (E) y los consecuentes de la conducta son analizados y no solo reducidos a variables ambientales. Otro de 


\section{PARADIGMAS EDUCATIVOS PARA UNA METOdOLOGÍA GLOBAL EN LA ENSEÑANZA}

Ios componentes más importantes dentro de este modelo es el estado biológico del organismo (0), el cual comprende a parte del propio estado puramente biológico del organismo, la variabilidad del bagaje, de la historia de aprendizaje y de las circunstancias que les haya tocado vivir a cada uno de los individuos, hasta ese momento. Por lo que podemos afirmar en base a Fernández-Ballesteros (2004) que "A medida que se incrementa la edad aumenta las diferencias existentes entre los individuos envejecientes debido, lógicamente, a la diversidad de circunstancias y de contextos históricos vividos" (Fernández-Ballesteros, 2004, p.1).

Para la realización de esta intervención se utilizan diferentes técnicas como: Técnicas para fomentar y mantener comportamientos adecuados. Dentro de estas encontramos cinco técnicas. La primera de estas es la del refuerzo positivo, la cual consiste en la introducción de un estímulo agradable, tras la realización de una conducta que se quiere fomentar o mantener. La segunda es el modelado, consistente en la imitación de la conducta observada, un claro ejemplo de esta técnica es el hecho de que, si a un alumno se le premia una acción o conducta, los demás compañeros que visualicen dicha conducta la tratarán de imitar para recibir ellos la recompensa también. En tercer lugar, nos encontramos con el modelamiento (shaping) que pretende reforzar las aproximaciones sucesivas. Técnica empleada para la construcción de nuevas conductas, a través de una conducta ya conocida. La técnica situada en cuarto lugar es el Principio de Premack, consistente en el uso de actividades preferidas por el sujeto como reforzadores de las actividades menos apreciadas por el mismo, un claro ejemplo de esta técnica es "si haces los deberes, vamos al parque." Para finalizar este apartado tenemos el contrato de contingencias, que consiste en un acuerdo escrito entre dos o más partes en el que se establecen las conductas que se han de realizar y las recompensas que pueden ser obtenidas.

Otros tipos de técnicas son para reducir o eliminar conductas inadecuadas. Comenzamos con el Castigo, técnica que se subdivide en dos, el castigo positivo, que consiste en la introducción de un estímulo no agradable tras la realización de una conducta indeseada, (copiar 100 veces "no pegaré a mi compañero") y el castigo negativo, consistente en la retirada o supresión de un estímulo placentero ("como has pegado a tu compañero, no saldrás al recreo hoy"). La segunda técnica que encontramos en este apartado es la saciedad, siendo esta el aumento de la cantidad de una recompensa hasta que el propio individuo desista de esta recompensa. En tercer lugar, tenemos el costo de respuesta, técnica que retira una serie de reforzadores ya obtenidos ("como has insultado a tu compañero, te retiro 0,5 puntos de comportamiento de clase de los 2 puntos que tenías"). La cuarta técnica que nos encontramos es la extinción, que consiste en la suspensión definitiva de una recompensa tras una conducta indeseada. Por último, tenemos la sobrecorrección, técnica consistente en la restitución del ambiente a un estado mucho mejor que el anterior, (como has pintado tu pupitre, ahora vas a limpiar los pupitres de todos tus compañeros").

\section{MÉTODO}

\section{Objetivos}

Para la realización de este trabajo, nos hemos centrado principalmente en elaborar una propuesta para trabajar la cohesión intragrupal y la mejora de la conducta de clase a partir de tres paradigmas de la psicología de la educación. Como segundo objetivo, realizar una primera aproximación fin conocer la cohesión de grupo de un curso de primer cuatrimestre de magisterio de educación primaria. 


\section{Diseño}

Esta investigación es descriptiva, etnográfica y no experimental. Según Castellanos (2020), este método de investigación, significa que este estudio tiene como finalidad definir, clasificar, catalogar o caracterizar el objeto de estudio. La etnografía se suele utilizar con frecuencia en el aula, utilizándose la observación participante, y tiene como objetivo "estudiar los acontecimientos que de manera natural ocurren en un grupo, con el fin de entender el significado que tales eventos tienen para las personas involucradas" (Castellanos, 2020).

\section{Usuarios}

Se trabaja dicha metodología con el grupo de clase de primer cuatrimestre de magisterio de educación primaria. Son alumnos que están recién ingresados a la universidad y el conocimiento como grupo no existe.

\section{Instrumentos Utilizados}

El instrumento utilizado es el sociograma, este puede definirse como "una técnica para determinar las preferencias de los individuos respecto a diversos estímulos (personas) que forman parte de su medio" (Calvo, 2010, p.1).

Un sociograma es un test que se realiza a un grupo y se compone de una serie de diferentes preguntas, de respuesta abierta. En este cuestionario se busca la reflexión más sincera de los sujetos y que éstos contesten a las preguntas buscando siempre a la 0 las personas que más asocian a la pregunta. Para esta investigación se plantean las siguientes preguntas: ¿Con qué tres compañeros te sentarías en un autobús si te fueses de excursión? y ¿con qué tres compañeros no te sentarías? o bien, en clase os mandan hacer un trabajo y quieres conseguir toda la nota posible, ¿a qué tres compañeros elegirías para hacer de tu grupo? Explica el por qué y a qué tres compañeros no escogerías. Aquí vemos que estas preguntas pueden oscilar tanto en ámbitos de ocio de la persona como en ámbitos de trabajo. Además, se realizan otras preguntas como: quién destaca por llevarse bien con los profesores, ser antipático con los demás, tener problemas para comunicarse, tener pocos amigos, ser simpático con los compañeros, estar dispuesto a ayudar a los demás, tener problemas para comunicarse y tener pocos amigos.

Tras la realización de este test, se obtienen diferentes datos como el grado de integración de cada sujeto, si algún sujeto es rechazado, la adaptabilidad que tienen los sujetos o características del individuo tales como si es o no amable con sus compañeros. En conclusión, un sociograma nos informará del clima existente en un grupo y las relaciones intragrupales que existen en él.

\section{Propuesta}

Antes de comenzar queremos aclarar que nuestro proyecto puede escalarse a diferentes niveles de educación primaria, secundaria y estudios superiores, siendo un profesor de una sola asignatura, siendo el maestro tutor de la clase, impartiendo varias asignaturas o pudiendo coordinarse con todo el equipo docente de la clase presentada para que todas las asignaturas recibidas por el alumnado sean impartidas del mismo modo. Comenzaremos la explicación basándonos que la propuesta es aplicada por los tutores de una clase. Nuestro proyecto comienza con la elaboración de un sociograma, que, con los resultados obtenidos de este, podremos comenzar con nuestro método. Primero, dividiremos a la clase en varios grupos de cinco personas, que podremos formar gracias a la interpretación de los resultados obtenidos del sociograma. Estos grupos se formarán de manera distinta para cada una de las asignaturas que nosotros impartimos, teniendo una duración de un trimestre, por lo que cada trimestre se realizará un nuevo sociograma, con el que podremos ir observando los cambios intragrupales que se van a ir dando a lo largo del curso académico. Con 


\section{PARADIGMAS EDUCATIVOS PARA UNA METOdOLOGÍA GLOBAL EN LA ENSEÑANZA}

esto conseguimos que sean los propios alumnos los que aprendan a trabajar en equipo, a ayudar a sus compañeros, mejorando así la cooperación y la convivencia, puesto que si uno del grupo adopta una conducta inadecuada afectará a la nota grupal. Además, con esta división conseguimos que ni un solo alumno se pueda quedar apartado del grupo clase, puesto que la idea es que todos los alumnos tengan que trabajar juntos alguna vez, y por tanto conseguir un objetivo común.

Para el desarrollo de la actividad del aula, hemos realizado una hipótesis de método pedagógico, en la cual nos basamos sobre tres paradigmas. En primer lugar, contamos con el paradigma conductista, concretamente en la parte de la modificación de conducta, ya que creemos que la escuela es un espacio de transformación social. La puesta en práctica de este apartado la vamos a hacer mediante la instauración de normas y reglas que los estudiantes han de seguir, por ejemplo, el buen comportamiento en clase, el respeto mutuo, el valor del esfuerzo o la constancia. Este comportamiento lo reforzaremos con la parte del condicionamiento operante, premiando 0 reforzando las conductas que queremos que sean repetidas o instauradas, y utilizando técnicas para disminuir conductas inadecuadas, las que queremos que desaparezcan. Este componente de nuestro proyecto se podrá ver reflejado en la evaluación como un $20 \%$ de la nota final, en el que podremos englobar, la participación en clase, la conducta del alumnado, interés por el aprendizaje y esfuerzo realizado. Este $20 \%$ será otorgado automáticamente al comienzo del trimestre a todo el alumnado, y estos lo tendrán que mantener cumpliendo las conductas que queremos reforzar, y eliminando las conductas que queremos extinguir. Este $20 \%$ se verá reducido cada vez que una de las conductas se vea perturbada, disminuyendo un $1 \%$ de la nota final cada vez que esto ocurra.

Respecto a la relación profesor alumno, hemos querido optar en este caso por una base cognitivo humanista, puesto que creemos que es muy importante que la relación entre ambos sea muy cercana, tanto para la mejora del propio aprendizaje, como para la convivencia en el aula, ya que si existe dicha relación los alumnos no tendrán ningún tipo de problema en poder acudir a su profesor, en este caso tutor, para contarle cualquier inconveniente que se haya producido tanto dentro como fuera del aula. Esta relación más estrecha le otorgará al alumnado la posibilidad de tener un apoyo de un adulto, puesto que pueden existir niños que provienen de familias desestructuradas, teniendo ciertas carencias de apoyo o afectividad (Sabroso, Jiménez y Lledó, 2011) siendo solventadas estas con dicha relación profesor alumno. Esto lo podemos observar en el propio día a día de la clase, así como en la manera de evaluación, como ya hemos explicado con anterioridad, los alumnos parten con un $20 \%$ de la nota adquirida al comienzo del curso, y por incumplir con las conductas deseadas, estos solo pueden ir reduciendo dicho porcentaje, nunca subirlo, pero para poder motivar al alumnado, y a su vez poder conseguir mayor esfuerzo por parte del mismo, planteamos la opción de que estos puedan recuperar dicha nota a través de la presentación de material complementario de la asignatura en cuestión, sin ningún tipo restricción, dándole total libertad al alumno en la elección del material a traer. De ese modo otorgamos al alumnado una manera de poder paliar conductas inadecuadas con esfuerzo, al mismo tiempo que aporta nuevos conocimientos o herramientas a toda la clase y al profesor, teniendo un aprendizaje bidireccional constante. Con esto intentaremos que la convivencia dentro y fuera del aula sea mucho más amena y mejor, teniendo menos problemas o que los que puedan surgir tengan una duración mucho más corta.

Para finalizar con los paradigmas sobre los que nos hemos basado para la realización de nuestra hipótesis, nos encontramos con el paradigma sociocultural o sociohistórico. De esta teoría hemos obtenido la parte en la que nos habla de las estrategias del aprendizaje, puesto que dividimos a la clase en grupos, tal y como defiende Vygotsky (1979), además de la sustitución de los deberes clásicos, por la asignación de trabajos grupales con una duración de una semana. Este cambio nos 
permite observar cada una de las zonas de desarrollo real y de desarrollo potencial que nos es planteada por el alumnado, teniendo un seguimiento continuo. Con este modus operandi hacemos que los propios alumnos vayan adquiriendo unas competencias, como la sociolingüística, puesto que han de exponer sus ideas al resto del equipo y debatirlas entre todos, con respeto y compañerismo. También podrán conocer las distintas maneras de trabajar que tendrán con cada uno de los distintos grupos a los que pertenecen en las asignaturas que nosotros como maestros tutores impartimos, siendo así mucho más versátiles y polivalentes. De esta manera también podemos mejorar la convivencia entre el alumnado, puesto que todos entre ellos van a tener que trabajar juntos en alguna asignatura, teniendo un objetivo común. En este tipo de organización podemos ver una base en el andamiaje que nos planteaba este paradigma, puesto que al tener un objetivo común han de ser ellos mismos los que se puedan apoyar, ayudar y comprender para poder alcanzarlo, aprendiendo de este modo los unos de los otros. En cuanto a la evaluación, estos trabajos semanales contarán un total de un $30 \%$ de la evaluación, teniendo estas varias connotaciones. Cada una de las presentaciones semanales que se hagan por cada grupo, tendrá una calificación objetiva del propio trabajo, que se podrá aumentar o disminuir para cada uno de los integrantes de dicho grupo en función de su esfuerzo elaborado para la realización del trabajo. En otras palabras, existirá una primera evaluación del trabajo en sí, sobre la que se procederá a una reevaluación individual para la obtención de la nota de este apartado de cada alumno. Para dicha reevaluación se procederá a preguntar al grupo y que sean los propios integrantes del grupo los que nos aclaren la cantidad de trabajo realizada por cada uno de ellos.

Para concluir con el apartado de la evaluación en el que ya tenemos un $20 \%$ de la misma, basada en la participación y conducta en clase, y un 30\% en los trabajos semanales, añadimos un 50\% que se obtendrá a través de un examen escrito. Hemos realizado esta división asimétrica del puntaje puesto que queremos que nuestros alumnos no vean a la escuela como un lugar donde te obligan a plasmar en un papel unos conocimientos carentes de cualquier tipo de comprensión, sino que queremos que los alumnos tengan una serie de competencias bien adquiridas y que sean capaces de poder entenderlas correctamente para llevarlas a la práctica. Consolidando así el 100\% de la nota final.

Como se ha podido observar a lo largo de todo el planteamiento, los alumnos se encuentran subdivididos en grupos la mayor parte del tiempo, teniendo nuestra hipótesis una carencia de cohesión de grupo clase. Por lo que para poder solventar dicha carencia planteamos lo siguiente. La creación de una identidad como grupo clase. Esta identidad se compone de:

1‥ Nombre de clase: Con la elección de un nombre de clase, creemos que aumentará en gran medida la cohesión de grupo, puesto que, bajo este nombre, todos los integrantes tendrán la misma cabida. Esta elección se llevará a cabo de forma que cada uno de los grupos propondrá un nombre a la clase, y una vez tengamos todos los nombres propuestos, será votado de forma individual, eligiendo el que más votos tenga.

2º. Logo: Para que la cohesión de grupo sea aún mayor, proponemos la creación de un logo de clase, un símbolo en el cual todos los integrantes puedan verse identificados. La elección de este logo se realizará de modo que el profesor planteará a los alumnos una serie de figuras o formas que se dividen en dos, la base y los ítems o complementos. Por ejemplo: Elegir una base: círculo, cuadrado, rectángulo o triángulo; elegir dos ítems: alas, gafas, sombrero y random. Un alumno voluntario dibujará el logo que todos los compañeros eligen. Estos elementos serán votados de forma grupal, teniendo que elegir una de las formas para la base, y dos de los ítems propuestos por grupo. Como en la anterior elección las figuras propuestas por los grupos serán votadas de manera individual. A la hora de configurar el logo con las figuras más votadas, se podrá ir modificando la 


\section{PARADIGMAS EDUCATIVOS PARA UNA METODOLOGÍA GLOBAL EN LA ENSEÑANZA}

posición, rotación y tamaño de los mismos entre todos, teniendo siempre respeto por los demás y de forma consensuada.

3․ Canción de clase: Para poder tener la mayor cohesión de grupo posible, creemos que la mejor manera de complementar el nombre y el logo de la clase, es tener una canción de clase. Un himno en el que todos los integrantes puedan identificarse, creando así la sensación de unidad buscada. Esta canción será escogida entre las propuestas realizadas por los distintos grupos, y votadas de manera individual, como ya se ha hecho con el nombre y logo. Esta canción será puesta por el maestro tutor al comienzo y fin de cada día lectivo.

\section{RESULTADOS}

Según el objetivo de esta investigación es elaborar una propuesta para trabajar la cohesión intragrupal y la mejora de la conducta de clase a partir de tres paradigmas de la psicología de la educación. Hemos visto anteriormente la propuesta, a continuación, se hace una primera aproximación a fin de conocer la cohesión grupal del curso de grado de magisterio de educación primaria, a través del uso del sociograma. En este grupo existen alumnos que son líderes destacándose por el color más oscuro, son alumnos que son los más elegidos por sus compañeros para trabajar o para actividades de ocio. Otros alumnos, con menor puntuación, son elegidos dentro de los grupos y son punto de conexión entre los compañeros. Destaca que hay dos alumnas que no son elegidas por el grupo y otros alumnos están en el exterior del sociograma que son elegidos por algún compañero, pero no hay una vinculación fuerte con el grupo (Figura 1).

Figura 1. sociograma anonimizado del grupo clase. Alumnos elegidos. Elaboración propia.

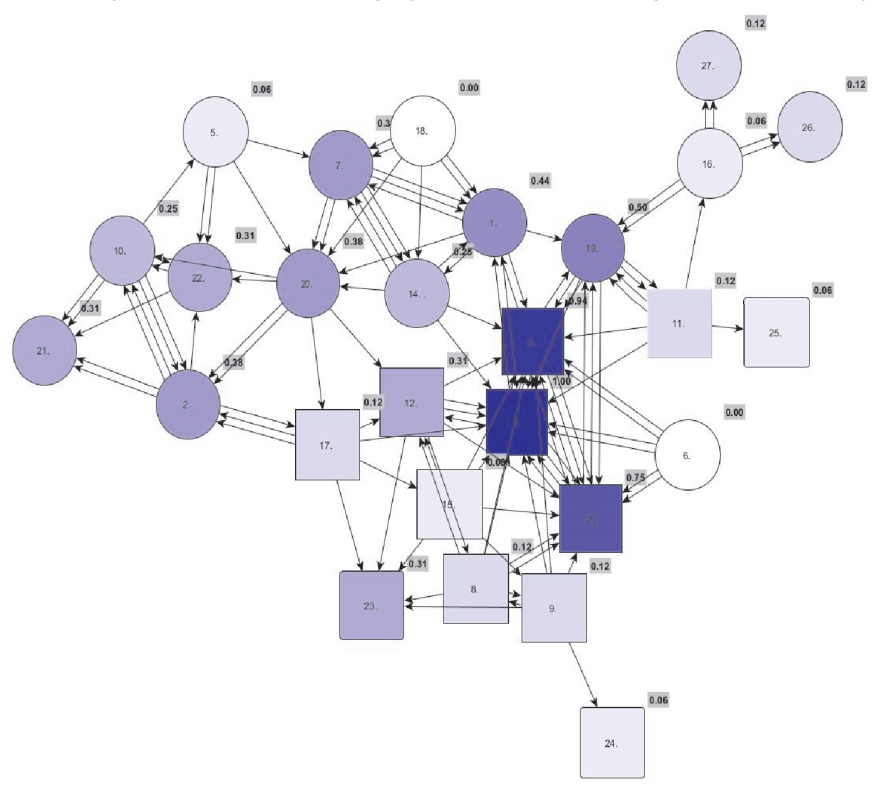

Podemos observar en la figura 2, el sociograma de los alumnos que no son elegidos por el grupo para compartir o para trabajar, coinciden con los alumnos que están más alejados del grupo y menos tenidos en cuenta según figura 1. Cabe destacar también que, aun siendo maestros de 
educación primaria, el tener una discapacidad presenta una característica de no elección, como sucede con la alumna número 6. Las alumnas 21 y 22 son acogidas por el grupo sin rechazo alguno, son alumnas que las tendrán en cuenta para trabajar o para compartir en actividades de ocio.

Figura 2. sociograma anonimizado del grupo clase. Alumnos no elegidos. Elaboración propia.

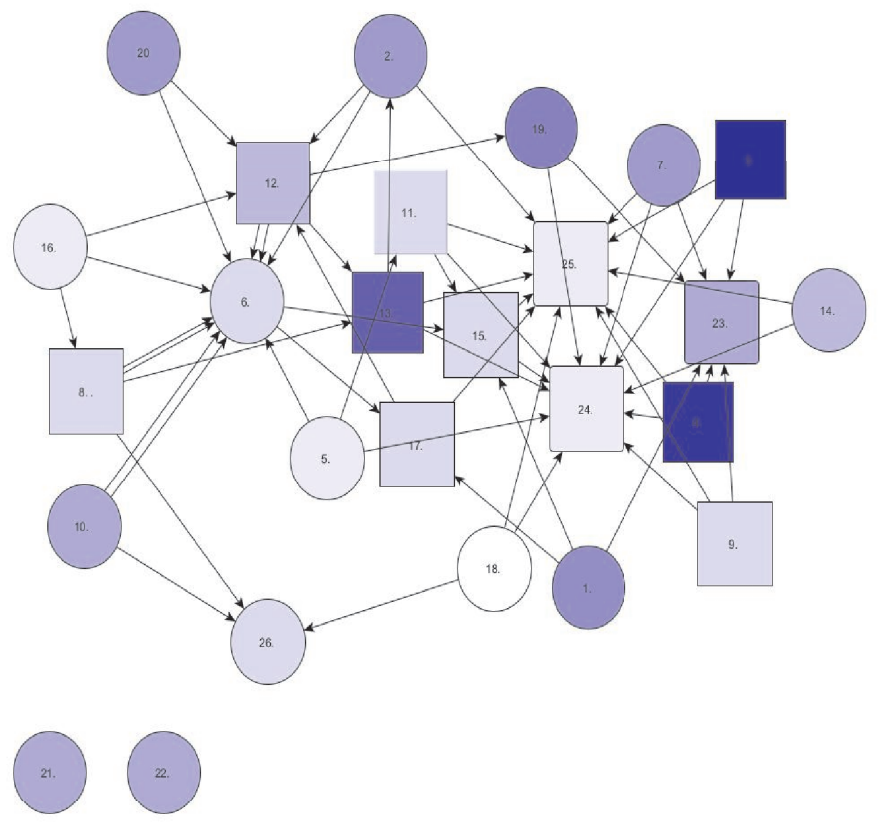

\section{CONCLUSIONES Y SUGERENCIA}

Esta investigación demuestra que es necesario abordar esta propuesta metodológica vista, para diluir los subgrupos cerrados, y para ayudar a los alumnos que son rechazados por su comportamiento durante el curso o por tener una característica especial, que de otra manera puede dar lugar a posibles conductas inadecuadas, desaprovechando las cualidades de los alumnos 0 arraigando los rechazos de algunos alumnos.

Con esta propuesta vemos que esta investigación pretende conseguir una buena cohesión de grupo, así como un buen camino sobre el que difundir el conocimiento al alumnado del presente y del futuro, pudiéndose llevar a cabo de manera sencilla gracias a la gran escalabilidad que esta hipótesis posee, de la que ya hemos hablado con anterioridad. Por todo esto, realizamos una propuesta que no es una dinámica efímera extendida en el tiempo, ya que se considera esencial trabajar la cohesión de grupo y más como futuros profesores, por lo que se desarrolla un proyecto dirigido para todo el año académico. Este se basa en la creación de una hipótesis de método pedagógico, que consta de una combinación de tres paradigmas teóricos, conductista, cognitivohumanista y cognitivo-sociocultural. Dentro del paradigma conductista, nos hemos fijado en la parte de la modificación de conducta a través del condicionamiento operante, además de en el tipo de evaluación. 


\section{PARADIGMAS EDUCATIVOS PARA UNA METOdOLOGÍA GLOBAL EN LA ENSEÑANZA}

También es necesario abordar, que del paradigma cognitivo-humanista hemos extraído la relación cercana entre el profesor y el alumno, creando así una confianza entre ellos. Del paradigma sociocultural, utilizaremos el andamiaje que se creará mediante los trabajos en grupo, se considera importante su evaluación antes, durante y después de las actividades realizadas. Para la realización de esta hipótesis metodológica, nos centraremos en la unión entre los alumnos de la clase y el profesor, siendo estos los protagonistas del proyecto anual propuesto. A través de la mejora de la conducta en clase, vemos que esta se puede extrapolar al ámbito familiar, creando así una transformación social que ayude a su situación en casa (Méndez, 2018). Cambiando de plano, tenemos al maestro tutor, que será la figura principal encargada de realizar esta metodología. Aparte, informaremos al resto de profesores que imparten clase a los alumnos del tipo de proyecto que queremos utilizar, y les propondremos unirse a este para mejorar la efectividad. De esta forma, podemos llegar a utilizar la misma forma de enseñanza para todas las asignaturas de la clase en cuestión. Si tras concluir el curso escolar, se reconoce el mérito de este proyecto y los resultados obtenidos son satisfactorios, se podría expandir esta metodología a todo el centro educativo, obteniendo así la mayor cantidad de recursos y una coordinación óptima. Desde nuestro punto de vista esta creación supone el hacer plausible un método con el que hubiéramos soñado recibir clase.

Existen diferentes investigaciones y artículos que discuten sobre las posibilidades de un método que combine distintos puntos y fundamentos de varias teorías educativas, creando una amalgama de metodología pedagógica funcional. Por ejemplo, Hernández Rojas, G. (1997) nos habla de que una de las diferencias entre la teoría conductista y la humanista se encuentra en que la primera se centra en los recursos para la modificación de conducta, mientras que la segunda se fija en el fin al que se quiere llegar. Pero de alguna forma esta distinción entre los medios y el fin se encarna en sugerencias de que el humanismo y el conductismo se pueden llegar a sintetizar combinando las técnicas conductistas con la filosofía humanista. Como está demostrado, la buena relación maestroalumno es fundamental para prevenir comportamientos no deseados, ya que se crea un clima agradable en el aula (Gómez y Cuña, 2017). Esto último, sería uno de los fundamentos clave de la teoría humanista, a la que se le puede añadir técnicas del método conductista, como la creación de unas normas explicadas de forma concisa y que los alumnos deben respetar, pudiendo según los casos, ser los propios alumnos los que ayuden a elaborar estas reglas, lo que les motivará y les concienciará aún más a seguirlas pues son ellos los que las han redactado (Gómez y Cuña, 2017). Como última investigación que también combina diferentes paradigmas nos encontramos con la tesis doctoral de Mg. César Segundo Llontop Sebastiani (2015), titulada: Aplicación del paradigma socio cognitivo humanista y el desempeño docente en las escuelas de educación superior del comando de educación y doctrina del ejército. Esta tesis nos muestra la unión entre los paradigmas sociocultural, cognitivos y el humanista, descartando por completo el paradigma conductista. Según Latorre (2010) "a partir de 1979, tras diversas lecturas y reflexiones llegué a la conclusión de que el paradigma conductista y sus aplicaciones al diseño curricular estaban ya agotados. En ese momento, dice él, tenía claro "lo que no era", pero "no sabía lo que era, y menos aún, cómo debía ser", a nivel teórico-práctico" (Latorre, 2010, p. 56 - 57). Desde nuestro punto de vista no concordamos con la idea que defiende este autor, considerando que del paradigma conductista también se pueden obtener métodos y recursos aplicables hoy en día, sin ser ni mucho menos esta una teoría obsoleta.

Finalmente, hacemos la petición de que aumenten este tipo de investigaciones y de aplicar este método, con el objetivo de poder tener la mayor cantidad de estudios que doten a los docentes de un mayor número de herramientas con las que puedan enseñar al alumnado del futuro. 


\section{REFERENCIAS BIBLIOGRÁFICAS}

Aragón, L. y Silva, A. (2008). Fundamentos teóricos de la evaluación psicológica. México: Pax.

Bertrán, J. (2019). Condicionamiento clásico o Plavloviano. En cómo aprenden los seres humanos (pp. 35-56). Ediciones UC. https://doi.org/10.2307/j.ctvkjb4jt.5

Bezanilla, J. (2011). Sociometría: Un método de investigación psicosocial. México: PEI

Castellanos Vega, R. (2020). Manual de psicología de la educación. Zaragoza: Digicopy.

Cava, G. (16 de mayo de 2014). Evaluación conductual. Análisis funcional. [Entrada en un blog]. https://online.ucv.es/resolucion/evaluacion-conductual-analisis-funcional-por-gloria-cava/

Fernández-Ballesteros, R. (2004). La psicología de la vejez. Encuentros multidisciplinares. Madrid: Biblioteca Nueva

Fingermann, H. (24 de agosto de 2012). Carl Rogers y la educación. [Entrada en un blog] https://educacion.laguia2000.com/general/carl-rogers-y-la-educacion

Francia, G. (12 de noviembre de 2020). Teorías cognitivas: cuáles son, tipos y ejemplos. PsicologíaOnline. https://Www.psicologia-online.com/teorias-cognitivas-cuales-son-tipos-y-ejemplos5321.html

González - Pérez, J. y Criado, M. (2010). Psicología de la educación para una enseñanza práctica. Madrid: CCS.

Gómez, M. y Cuña, A. (2017). Estrategias de intervención en conductas disruptivas. Educação Por Escrito, 8(2), 278-293. https://doi.org/10.15448/2179-8435.2017.2.27976

Hernández Rojas, G. (1997). Caracterización del Paradigma Humanista. Módulo Fundamentos Del Desarrollo de La Tecnología Educativa, 1-12. Recuperado de https://comenio.files.wordpress.com/2007/08/paradigma_humanista.pdf

Lima, L., Barbosa, Z., y Peixoto, S. (2018). Teoría humanista: Carl Rogers e a educação. Caderno de Graduação-Ciências Humanas e Sociais, 4(3), 161-172.

Llontop Sebastiani, M. (2015). Aplicación del paradigma socio cognitivo humanista y el desempeño docente en las escuelas de educación superior del comando de educación y doctrina del ejército. (Doctor). Universidad Nacional de Educación Enrique Guzmán y Valle.

Méndez, M (2018). La influencia familiar en la construcción de la identidad personal. Fundamentos y métodos para la formación permanente de los maestros de Educación Infantil. Análisis y prospectiva en la CAM. Madrid: Universidad Complutense de Madrid.

Myers, D. G. (2000). Psicología. Madrid: Editorial Médica Panamericana.

Morales, H y Irigoyen, A. (2017). El paradigma conductista y constructivista de la educación a través del decálogo del estudiante. Archivos En Medicina Familiar, 18(2), 27-30. Recuperado de https://www.medigraphic.com/pdfs/medfam/amf-2016/amf162a.pdf

Real Academia Española. (2001). Líder. En Diccionario de la lengua española (23.a ed.). Recuperado de $h$ ttps://dle.rae.es/lider

Ruiz Carrillo, E. y Estrevel Rivera, L. B. (2010). Vigotsky: la escuela y la subjetividad. Pensamiento psicológico, 8 (15), 135-145.

Sabroso, A., Jiménez, M. D., Lledó, A. (2011). Problemas familiares generadores de conductas disruptivas en alumnos. International Journal of Developmental and Educational Psychology. INFAD revista de Psicología, 1 (2), 423-432

Vielma Vielma, E., Salas, M. L. (2000). Aportes de las teorías de Vygotsky, Piaget, Bandura y Bruner. Paralelismo en sus posiciones en relación con el desarrollo. Educere, 3 (9), 30-37.

Vygotsky, L. (1979). El desarrollo de los procesos psicológicos superiores. Buenos Aires: Grijalbo

Villamizar, L. J. (2017). The effects of Vygotsky's sociocultural theory on second language acquisition and language Input. Espiral, Revista de Docencia e Investigación., 7(1), 91-102. Recuperado de http://revistas.ustabuca.edu.co/index.php/ESPIRAL/article/view/178 


\section{PARAdigmaS EdUCATIVOS PARA UNA METOdOLOGíA GLOBAL EN LA ENSEÑANZA}

Villón Tomalá, A. M. (2017). Sociograma escolar, una técnica eficaz. Illari, (3), 39-44. Recuperado a partir de https://revistas.unae.edu.ec/index.php/illari/article/view/246 